\title{
Impact of inter-lab variation on the estimation of epidemiological cut-off values for disc diffusion susceptibility test data for Aeromonas salmonicida
}

\author{
PeteSmith ${ }^{\mathrm{a}}$, Neil M. Ruane ${ }^{\mathrm{b}}$, Iain Douglas ${ }^{\mathrm{a}}$, Cyril Carroll ${ }^{\mathrm{a}}$, Göran Kronvallc, \\ Gerard T.A. Fleming a \\ ${ }^{a}$ Department of Microbiology, National University of Ireland Galway, Ireland \\ ${ }^{\mathrm{b}}$ Fish Health Unit, Marine Institute, Oranmore, Galway, Ireland \\ ${ }^{c}$ Department of Microbiology and Tumor Biology-MTC, Clinical Microbiology, Karolinska Institute, Stockholm, Sweden
}

\begin{abstract}
Two laboratories investigated the susceptibility of 106 Aeromonas salmonicida strains (from Denmark, France, Ireland, Norway and Scotland) to erythromycin, gentamicin, oxytetracycline and oxolinic acid using the disc diffusion protocols (M42-A) published by the Clinical and Laboratory Standards Institute. In studies of susceptibility to florfenicol an additional 15 Canadian strains were included. Comparison of the data generated by the two laboratories demonstrated that for each disc both detected a similar pattern of distribution but that there was a significant numerical difference in the zone sizes they recorded. Analysis of the extent of this lateral shift between the data generated in two laboratories indicated that the application of a single laboratory-independent epidemiological cut-off value for each disc could result in disagreement between the laboratories as to whether a strain should be classified as wild-type or non wild-type.

Normalised resistance interpretation was employed to generate epidemiological cut-off values from the data obtained by each laboratory. The use of these laboratory-specific cut-off values resulted in both laboratories achieving complete agreement as to the classification of all strains to all agents.
\end{abstract}

\section{Introduction}

A recent survey (Smith, 2006) indicated that the majority of laboratories, involved in investigating the susceptibility of clinical isolates from fish diseases, use disc diffusion methods. This survey also presented evidence of an increasing harmonisation of the disc diffusion protocols being employed, with the majority of the responding laboratories using either the earlier versions of CLSI M42-A (CLSI, 2006a) or the functionally equivalent protocols published by Alderman and Smith (2001). However, the survey also revealed that, despite the frequent use of similar test protocols, there was a considerable variation between laboratories with respect to the breakpoints they applied 
to the data they obtained. The current challenge is, therefore, to develop and validate a set of breakpoints that will allow the attribution of clinically relevant meaning to the inhibition zone data these laboratories generate.

Clinically relevant breakpoints should provide criteria by which the data generated by testing any isolate could be used to determine whether, under the conditions of a proposed therapy, that strain should be considered as sensitive or resistant. Thus, in addition to data on the susceptibility of the organism, the setting of such breakpoints would require data on the pharmacokinetic and pharmacodynamic (PK/PD) aspects of the proposed therapy and on the clinical efficacy of previous therapies (MacGowan and Wise, 2001; CLSI, 2002; Kahlmeter et al., 2003). Even when all these data are available, Kahlmeter et al. (2003) have argued that the setting of clinical breakpoints is not and probably never will be, exact or strictly scientific.

With respect to orally administered treatments of populations of poikilothermic animals, the acquisition of all the PK/PD data that would be required would present a number of theoretical (Coyne et al. 2004a,b, 2006) and practical (Samuelsen, 2006) problems and would require a significant period of time (Smith, 2001). In contrast, data on the distributions of susceptibility for any particular group of bacteria associated with fish disease are relatively simple to accumulate. The European Committee on Antimicrobial Susceptibility Testing (EUCAST) (EUCAST, 2000) and CLSI (2002) have recommend that such distribution data can be used to estimate epidemiological cut-off values. These cut-off values can then be applied to the characterisation of isolates as wild type (WT) or non-wild type (NWT) on the basis of their relative susceptibility to a specific agent (EUCAST, 2000).

Miller and Reimschuessel (2006) have used the Clinical and Laboratory Standards Institute (CLSI) standard protocols (CLSI, 2006a,b) to measure the susceptibility of 217 strains of Aeromonas. salmonicida to oxytetracycline, florfenicol, oxolinic acid and ormetoprim-sulfadimethoxine. They used these zone size and minimum inhibitory concentration (MIC) data to estimate epidemiological cut-off values (Kahlmeter et al., 2003; CLSI, 2002) for this species. Kahlmeter et al. (2003) have argued that, in the absence of clinical breakpoints, epidemiological cut-off values of the type established by Miller and Reimschuessel (2006) can be used to detect and monitor resistance.

A central problem for any attempt to set universally applicable breakpoints or epidemiological cut-off values for disc diffusion data derives from the extent of the inter-laboratory variation that is associated with the data generated by this method (Kronvall, 1982, 2003; Kronvall et al., 1988). The development and application of strictly specified standard test protocols such as those recently published for bacteria isolated from aquatic animals (Alderman and Smith, 2001; CLSI, 2006a) aims to reduce the extent of this variation. However, increasing standardisation has been demonstrated only to minimise but not eliminate inter-laboratory variance (Kronvall, 2003). Inter-laboratory studies have been published of the data generated by three disc diffusion test protocols designed to examine bacteria associated with aquatic animals (Miller et al., 2003; NicGabhainn et al., 2003; Huys et al., 2005). All have reported significant inter-laboratory variance between the data generated by laboratories using the same test protocols. The width of the acceptable ranges for control strains in M42-A (CLSI, 2006a), which were generated from the standard deviations of data generated in nine laboratories (Miller et al. 2003), illustrate the extent to which inter-laboratory variation remains a problem even after rigorous standardisation. For A. salmonicida ACTC 33658 incubated at $22^{\circ} \mathrm{C}$ for $44-48 \mathrm{~h}$ the width of these acceptable ranges vary from 11-16 mm (median $13 \mathrm{~mm}$ ).

Some workers have responded to the extent of interlaboratory variation in disc diffusion data by abandoning any attempt to develop laboratory-independent, 'universal' breakpoints for data generated by standard disc diffusion protocols. As an alternative, these workers (Kronvall et al. 1988; Manninen et al. 1995, 1998; Kronvall et al., 2003) have developed a variety of methods of producing valid but laboratory-specific, breakpoints. Single strain regression analysis (Forsberg et al. 1985; Kronvall and Ringertz, 1991), standard curve regression (Kronvall et al., 1988) and two-strain linear regression (Manninen et al., 1998) have all been suggested as methods of generating laboratory- and species-specific disc diffusion breakpoints. All these approaches, however, rely on the prior existence of a consensus MIC breakpoint.

More recently Kronvall and his co-workers (Joneberg et al., 2003; Kronvall, 2003; Kronvall et al, 2003) have developed a method of generating interpretative criteria for disc diffusion data that are both species- and laboratory-specific but that do not rely on the prior existence of a consensus MIC breakpoint. In essence this method, normalized resistance interpretation (NRI), approaches the setting of breakpoints from a consideration of the mean and standard deviation of the distribution of zone sizes obtained, for a set of susceptible (WT) strains of a particular species, in any 
particular laboratory. A core feature of NRI is that it calculates the parameters of the normal distribution of susceptible strain zone sizes from the high-zone end of the experimentally determined distributions. This allows NRI to characterise the susceptible strain data distributionin a way that is notinfluenced or distorted by zones generated from strains with low-levels of resistance.

The work reported here was undertaken to determine the influence of inter-laboratory variations in the determination of epidemiological cut-off values for disc diffusion data generated using the M42-A (CLSI, 2006a) protocols. Given the fact that, with respect to bacteria associated with diseases of aquatic animals, no breakpoint MIC values have yet been established and the relatively urgent need for more rational interpretative criteria for disc diffusion data (Smith, 2006), this work was also designed to investigate the value of the NRI approach to the setting of laboratory specific epidemiological cut-off values.

\section{Materials and methods}

\subsection{Laboratories}

The work reported here was performed in the Fish Health Unit laboratory of the Marine Institute, Oranmore, Galway Ireland (Laboratory 1) and that of the Fish Disease Group, Department of Microbiology, National University of Ireland Galway, Ireland (Laboratory 2).

\subsection{Bacteria}

In the studies with erythromycin (ERY), gentamicin (GEN), oxolinic acid (OXA) and oxytetracycline (OTC) 106 strains of $A$. salmonicida were investigated. These strains were received from Scotland (33 strains), Norway (30 strains), Denmark (17 strains), France (15strains) and Ireland (11 strains). In the studies with florfenicol (FLO) and additional 15 strains obtained from Canada were also included. All strains were brown pigmented and reacted with antiserum (BioNor MONO AQUA).

Both laboratories obtained and maintained independent cultures of the control strain $A$. salmonicida NCIMB 1102 (equivalent to ACTC 33658) from the NCIMB (Aberdeen, UK).

\subsection{Media}

In both laboratories, bacteria were routinely cultured using Mueller Hinton Agar or Mueller Hinton Broth purchased from Mast Group (Bootle, UK). Incubations were performed at $22 \pm 2{ }^{\circ} \mathrm{C}$ for $44-48 \mathrm{~h}$.

\subsection{Discs}

Antimicrobial agent discs containing $15 \mu$ g erythromycin $\left(E R Y_{15}\right), 10 \mu \mathrm{g}$ gentamicin $\left(\mathrm{GEN}_{10}\right), 30 \mu \mathrm{g}$ florfenicol $\left(\mathrm{FLO}_{30}\right)$, and $30 \mu \mathrm{g}$ oxytetracycline $\left(\mathrm{OTC}_{30}\right)$ were obtained from Oxoid (Basingstoke, UK). Laboratory 1 obtained their $2 \mu \mathrm{g}$ oxolinic acid $\left(\mathrm{OXA}_{2}\right)$ from Mast Group (Bootle, UK) and Laboratory 2 obtained the same discs from Oxoid (Basingstoke, UK).

\subsection{Diffusion assay}

All disc diffusion assays were performed according to the protocols specified in M42-A (CLSI, 2006a). Suspensions were made in saline and incubations were at $22 \pm 2{ }^{\circ} \mathrm{C}$ and zones were read after $44-48 \mathrm{~h}$. A. salmonicida NCIMB 1102 was used as the control strain.

\subsection{Statistical analysis}

Normalised resistance interpretation was performed according to the method of Kronvall (2003) and Kronvall et al (2003). Peak values were established using 5 point rolling means and the mean and standard deviation of the real distribution of fully susceptible (wild type) strains were calculated using a plot of probit values against zone size. In each case 6 probit values were plotted and these included the first value $\mathrm{N} 5$ and the next five values lower than this. Epidemiological cut-off values were set at two and a half standard deviations below the mean.

All other statistical analyses were performed using InStat3.0 (GraphPad Inc).

\section{Results}

\subsection{Compliance with acceptable ranges for control strain}

During this work, both laboratories performed 9 independent measurements of the zones generated by each of the five discs against the control strain A. salmonicida NCIMB 1102. With respect to ERY, $\mathrm{GEN}_{10}, \mathrm{FLO}_{30}$ and $\mathrm{OXA}_{2}$ all 72 individual measurements lay within the acceptable ranges specified in M42-A (CLSI, 2006a). For OTC 30 all control strain zones determined by Laboratory 1 also lay within the acceptable range but the majority of the zones recorded by Laboratory 2 were larger than the upper limit of the acceptable range. Table 1 presents the mean zone sizes recorded by both laboratories. For Laboratory 1 the 
Table1

Comparison of the values $(\mathrm{mm})$ reported by each laboratory for the control strain mean $(n=9)$ and for the mean and epidemiological cutoff values generated for fully susceptible (WT) strains by NRI analysis

\begin{tabular}{|c|c|c|c|c|c|}
\hline \multirow[t]{2}{*}{ Disc } & \multirow[t]{2}{*}{$\begin{array}{l}\text { Acceptable } \\
\text { range } \\
(\mathrm{M} 42-\mathrm{A})\end{array}$} & \multirow[t]{2}{*}{ Lab } & \multirow{2}{*}{$\begin{array}{l}\text { Control } \\
\text { strain }\end{array}$} & \multicolumn{2}{|c|}{$\begin{array}{l}\text { NRI values for fully } \\
\text { susceptible } \\
\text { (WT) strains }\end{array}$} \\
\hline & & & & Mean & $\begin{array}{l}\text { Epidemiological } \\
\text { cut-of value }\end{array}$ \\
\hline \multirow[t]{3}{*}{ ERY $15 \mu \mathrm{g}$} & \multirow[t]{3}{*}{$19-31$} & 1 & 21 & 19 & 10 \\
\hline & & 2 & 25 & 23 & 14 \\
\hline & & $\begin{array}{l}\text { Lateral } \\
\text { shift* }\end{array}$ & 4 & 4 & 4 \\
\hline \multirow[t]{3}{*}{ FLO $30 \mu \mathrm{g}$} & \multirow[t]{3}{*}{$34-47$} & 1 & 36 & 36 & 27 \\
\hline & & 2 & 41 & 41 & 31 \\
\hline & & $\begin{array}{l}\text { Lateral } \\
\text { shift }\end{array}$ & 5 & 5 & 4 \\
\hline \multirow[t]{3}{*}{ GEN $10 \mu \mathrm{g}$} & \multirow[t]{3}{*}{$22-32$} & 1 & 22 & 22 & 14 \\
\hline & & 2 & 26 & 26 & 19 \\
\hline & & $\begin{array}{l}\text { Lateral } \\
\text { shift }\end{array}$ & 4 & 4 & 5 \\
\hline \multirow[t]{3}{*}{ OTC $30 \mu \mathrm{g}$} & \multirow[t]{3}{*}{$28-38$} & 1 & 32 & 34 & 25 \\
\hline & & 2 & 40 & 41 & 33 \\
\hline & & $\begin{array}{l}\text { Lateral } \\
\text { shift }\end{array}$ & 8 & 7 & 8 \\
\hline \multirow[t]{3}{*}{ OXA $2 \mu \mathrm{g}$} & \multirow[t]{3}{*}{$33-45$} & 1 & 35 & 38 & 30 \\
\hline & & 2 & 39 & 41 & 33 \\
\hline & & $\begin{array}{l}\text { Lateral } \\
\text { shift }\end{array}$ & 4 & 3 & 3 \\
\hline
\end{tabular}

Lateral shift was calculated as the difference $(\mathrm{mm})$ between the values calculated by the two laboratories.

All values were rounded off to the nearest $\mathrm{mm}$ before calculating lateral shift.

mean values recorded for all agents were close to the lowest acceptable value. With the exception of those generated for $\mathrm{OTC}_{30}$ the control zones reported by Laboratory 2 were closer to, but always smaller than, the mid-point of the acceptable range.

\subsection{Estimations of epidemiological cut-off values}

\subsubsection{Erythromycin}

Fig. 1a presents a scatter plot of the zone sizes generated by the two laboratories using discs containing $15 \mu \mathrm{g}$ erythromycin. The zones recorded for all 106 strains fell into a single cluster and no major discrepancies were observed between the data generated by the two laboratories for any single strain. Fig. 1b presents the same data as a histogram. For the data from Laboratory 1 the plot of the probit values against zone size was linear $\left(r_{2}=0.994\right)$ and the mean and standard deviations were $18.9 \pm 3.6$ Thus, for these data the NRI analysis indicated a cut-off value of $10 \mathrm{~mm}$ (Fig. 1a and b). For the data from Laboratory 2, the plot of the probit values against zone size was linear $\left(r_{2}=0.992\right)$ and the mean and standard deviations were $22.7 \pm 3.3 \mathrm{~mm}$. Thus, for these data the NRI analysis indicated a cut-off value of $14 \mathrm{~mm}$ (Fig. 1a and b). The application of these cutoff values resulted in both laboratories categorising all 106 strains as WT.

\subsubsection{Florfenicol}

Fig. 2a presents a scatter plot of the 121 paired zone sizes generated by the two laboratories using $\mathrm{FLO}_{30}$ discs. No major discrepancies were observed between the data generated by the two laboratories for any single

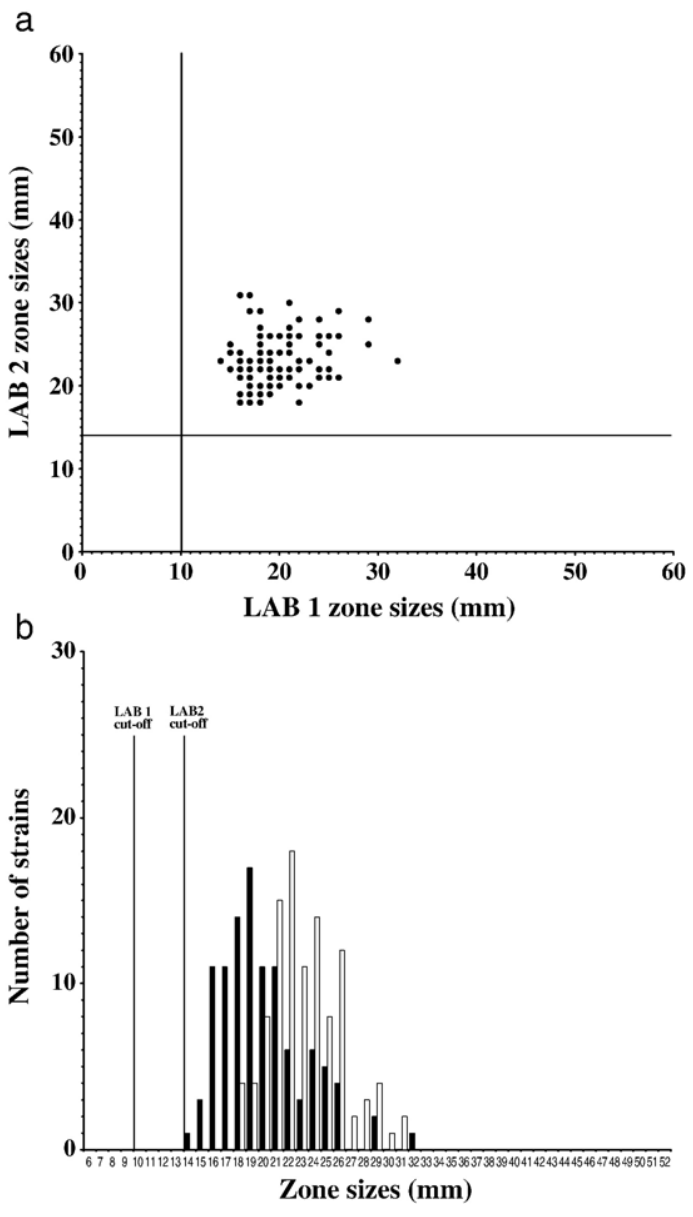

Fig. 1. a. Scatter-plot of the zones recorded by Laboratories 1 and 2 for discs containing $15 \mu \mathrm{g}$ erythromycin against 106 strains of A. salmonicida. Vertical line indicates the epidemiological cut-off value calculated by Laboratory 1 and the horizontal line that for Laboratory 2. b. Zones obtained by Laboratories 1 and 2 for discs containing $15 \mu$ g erythromycin against 106 strains of $A$. salmonicida. Solid bars indicate data from Laboratory 1 and open bars those for Laboratory 2 . The epidemiological cut-off values calculated by the two laboratories are shown as vertical lines. 

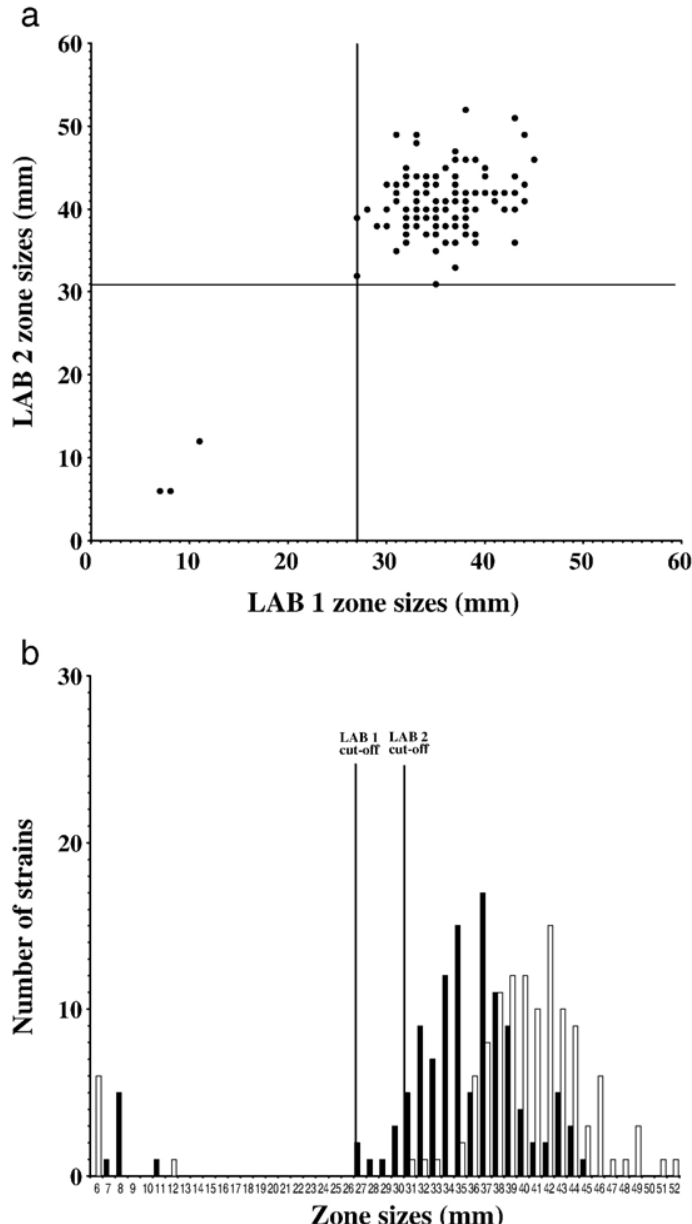

Fig. 2. a. Scatter-plot of the zones recorded by Laboratories 1 and 2 for discs containing $30 \mu \mathrm{g}$ florfenicol against 121 strains of $A$. salmonicida. Vertical line indicates the epidemiological cut-off value calculated by Laboratory 1 and the horizontal line that for Laboratory 2. b. Zones obtained by Laboratories 1 and 2 for discs containing $30 \mu \mathrm{g}$ florfenicol against 121 strains of $A$. salmonicida. Solid bars indicate data from Laboratory 1 and open bars thosefor Laboratory 2. Theepidemiological cut-off values calculated by the two laboratories are shown as vertical lines.

strain. Fig. $2 b$ presents the same data as a histogram. For the data from Laboratory 1 the plot of the probit values against zone size was linear $\left(r_{2}=0.984\right)$ and the mean and standard deviations were $36.4 \pm 3.7$. Thus, for these data the NRI analysisindicated a cut-off value of $27 \mathrm{~mm}$ (Fig. 2a and b). For the data from Laboratory 2, the plot of the probit values against zone size was linear $\left(r_{2}=0.989\right)$ and the mean and standard deviations were $40.9 \pm 3.8 \mathrm{~mm}$. Thus, for these data the NRI analysis indicated a cut-off value of $31 \mathrm{~mm}$ (Fig. 2a and b). The application of these cut-off values resulted in both laboratories categorising 6 strains, all originating from Canada, as NWT and the remaining 115 strains as WT.

\subsubsection{Gentamicin}

Fig. 3a presents a scatter plot of the zone sizes generated by the two laboratories using $\mathrm{GEN}_{10}$ discs containing. The zones recorded for all but one of the 106 strains fell into a single cluster and no major discrepancies were observed between the data generated by the two laboratories for any single strain. Fig. 3b presents the same data as a histogram. For the data from Laboratory 1 the plot of the probit values against zone size was linear $\left(r_{2}=0.941\right)$ and the mean and standard deviations were $22.3 \pm 3.4 \mathrm{~mm}$. Thus, for these data the NRI analysis indicated a cut-off value of $14 \mathrm{~mm}$ (Fig. 3a
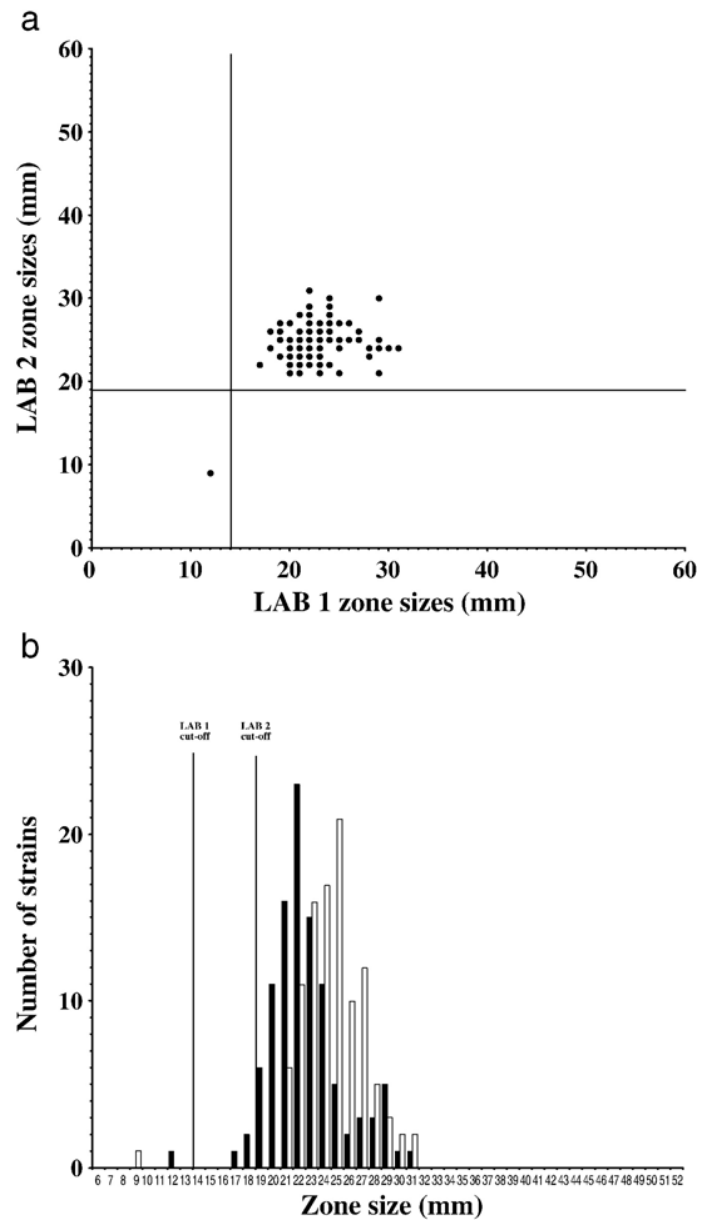

Fig. 3. a. Scatter-plot of the zones recorded by Laboratories 1 and 2 for discs containing $10 \mu \mathrm{g}$ gentamicin against 106 strains of A. salmonicida. Vertical line indicates the epidemiological cut-off value calculated by Laboratory 1 and the horizontal line that for Laboratory 2. b. Zones obtained by Laboratories 1 and 2 for discs containing $10 \mu \mathrm{g}$ gentamicin against 106 strains of A. salmonicida. Solid bars indicate data from Laboratory 1 and open bars those for Laboratory 2 . The epidemiological cut-off values calculated by the two laboratories are shown as vertical lines. 

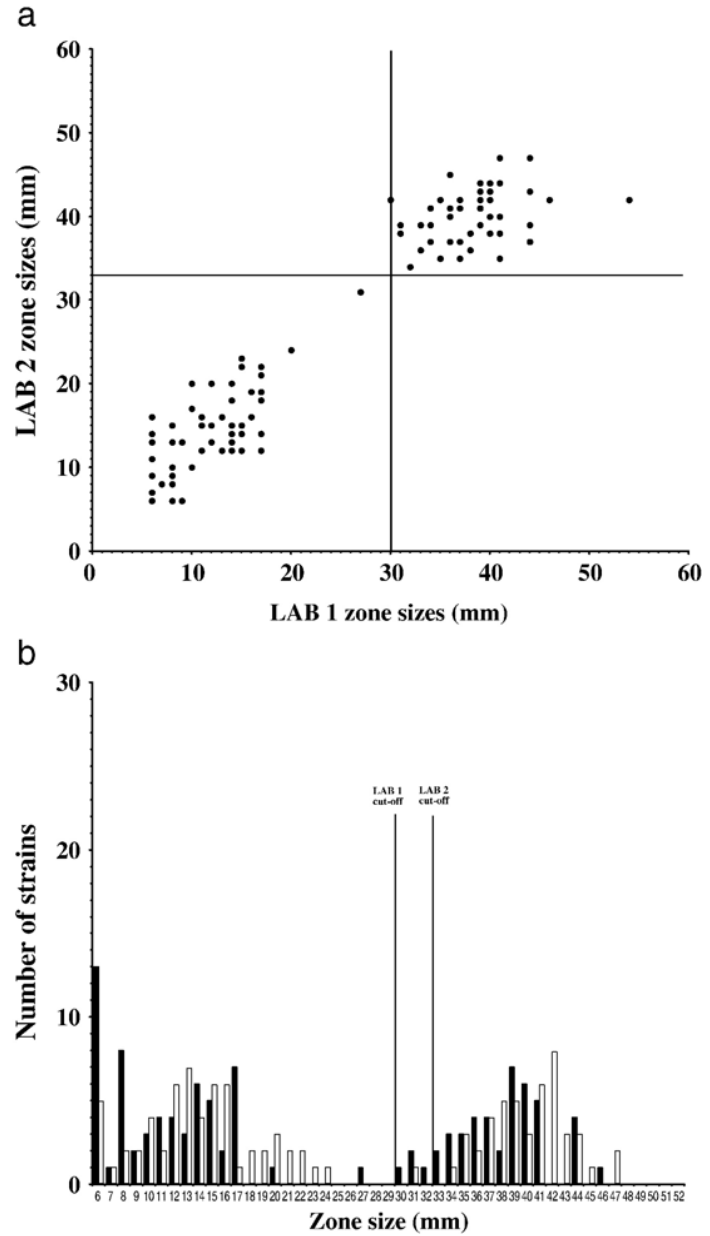

Fig. 4. a. Scatter-plot of the zones recorded by Laboratories 1 and 2 for discs containing $2 \mu \mathrm{g}$ oxolinic acid against 106 strains of $A$. salmonicida. Vertical line indicates the epidemiological cut-off value calculated by Laboratory 1 and the horizontal line that for Laboratory 2. b. Zones obtained by Laboratories 1 and 2 for discs containing $30 \mu \mathrm{g}$ oxytetracyclineagainst 106 strains of A. salmonicida. Solid bars indicate data from Laboratory 1 and open bars those for Laboratory 2 . Theepidemiological cut-off values calculated by the two laboratories are shown as vertical lines.

and $b)$. For the data from Laboratory 2 , the plot of the probit values against zone size was linear $\left(r_{2}=0.985\right)$ and the mean and standard deviations were $25.5 \pm$ $2.5 \mathrm{~mm}$. Thus, for these data, the NRI analysisindicated a cut-off value of $19 \mathrm{~mm}$ (Fig. 3a and b). Using the cutoff values calculated from their data, both laboratories classified one strain, originating in Scotland, as NWT and the other 105 as WT.

\subsubsection{Oxolinic acid}

Fig. 4a presents a scatter plot of the zone sizes generated by the two laboratories using $\mathrm{OXA}_{2}$ discs. No major discrepancies were observed between the data generated by the two laboratories for any single strain. Fig. $4 \mathrm{~b}$ presents the same data as a histogram. For the data from Laboratory 1 the plot of the probit values against zone size was linear $\left(r_{2}=0.961\right)$ and the mean and standard deviations were $38.4 \pm 3.2 \mathrm{~mm}$. Thus, for these data, the NRI analysis indicated a cut-off value of $30 \mathrm{~mm}$ (Fig. 4a and b). For the data from Laboratory 2, the plot of the probit values against zone size was linear $\left(r_{2}=0.984\right)$ and the mean and standard deviations were $40.5 \pm 2.9 \mathrm{~mm}$. Thus, for these data, the NRI analysis
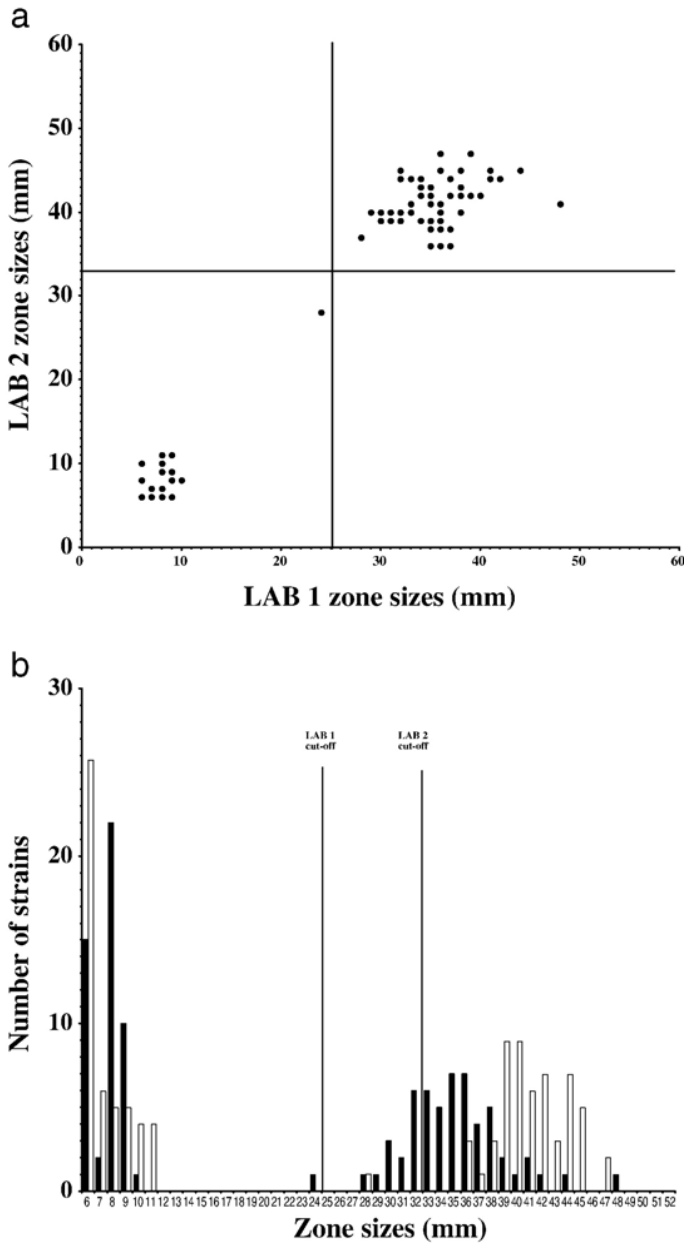

Fig. 5. a. Scatter-plot of the zones recorded by Laboratories 1 and 2 for discs containing $30 \mu \mathrm{g}$ oxytetracycline against 106 strains of A. salmonicida. Vertical line indicates the epidemiological cut-off value calculated by Laboratory 1 and the horizontal line that for Laboratory 2. b. Zones obtained by Laboratories 1 and 2 for discs containing $30 \mu \mathrm{g}$ oxytetracycline against 106 strains of A. salmonicida. Solid bars indicate data from Laboratory 1 and open bars those for Laboratory 2 . The epidemiological cut-off values calculated by the two laboratories are shown as vertical lines. 
indicated a cut-off value of $33 \mathrm{~mm}$ (Fig. $4 \mathrm{a}$ and b). Using the cut-off values calculated from their data both laboratories classified 60 strains as NWT and 46 strains as WT.

\subsubsection{Oxytetracycline}

Fig. 5a presents a scatter plot of the zone sizes generated by the two laboratories using $\mathrm{OXA}_{2}$ discs. No major discrepancies were observed between the data generated by the two laboratories for any single strain. Fig. $5 \mathrm{~b}$ presents the same data as a histogram. For the data from Laboratory 1 the plot of the probit values against zone size was linear $\left(r_{2}=0.995\right)$ and the mean and standard deviations were $34.4 \pm 3.8 \mathrm{~mm}$. Thus, for these data the NRI analysis indicated a cut-off value of $25 \mathrm{~mm}$ (Fig. 5a and b). For the data from Laboratory 2, the plot of the probit values against zone size was linear $\left(r_{2}=0.987\right)$ and the mean and standard deviations were $41.2 \pm 3.4 \mathrm{~mm}$. Thus, for these data the NRI analysis indicated a cut-off value of $33 \mathrm{~mm}$ (Fig. $5 \mathrm{a}$ and b). Using the cut-off values calculated from their data both laboratories classified 51 strains as NWT and 55 strains as WT. Fifty of the NWT strains gave zones in a tight cluster but one strain gave zones intermediate between this cluster and those generated by the other WT strains.

\section{Discussion}

\subsection{Strategies for setting epidemiological cut-off values}

One strategy for setting disc diffusion cut-off values for bacteria associated with fish disease is to attempt to deal with the problem of inter-laboratory variation by developing a rigorously specified standard protocol. This strategy underlay the development of the Alderman and Smith (2001) protocols and the M42-A protocols of the CLSI (2006a). For Group 1 bacteria such as $A$. salmonicida, these protocols are functionally equivalent but the inclusion, by M42-A, of acceptable ranges for control strains represents a valuable development. The assumption was that epidemiological cut-off values could be generated (Miller and Reimschuessel, 2006) that could be treated as 'universal' in that they could be validly applied by any laboratory that was in compliance with the acceptable ranges specified in M42-A (CLSI, 2006a).

An alternative strategy to setting breakpoints starts from the assumption that inter-laboratory variation is significant and cannot adequately be dealt with by increasing the rigour with which disc diffusion protocols are specified (Kronvall, 1982). Thus, this approach does not aim at the generation of 'universal' epidemiological cut-off values but adopts a strategy aimed at developing a universal method for setting laboratory-specific epidemiological cut-off values.

One of the key factors in making a decision between these two strategies will be the extent to which interlaboratory variation in the performance of disc diffusion tests (NicGabhainn et al., 2003) result in significant differences, lateral shift, between the numerical data obtained by different laboratories examining the same strain set.

\subsection{Evidence for lateral shift in distributions}

In this work, two laboratories, using the same standard disc diffusion protocol to examine the same strain set, produced data that showed very similar distribution patterns (Figs. 1a, 2a, 3a, 4a and 5a). Figs. $1 b, 2 b, 3 b, 4 b$ and $5 b$, however, demonstrate that, for all five discs, there was a significant ( $t$-test; $p b 0.05$ ) difference (data not shown) in the numerical values of the zone sizes recorded by the two laboratories. Table 1 presents the impact of these differences on the means recorded for the control strain and for the means and cut-off values estimated for WT strains by NRI analysis by the two laboratories. This suggests that, for any specific disc, lateral shift, the numerical difference in the data produced by two laboratories, is a parameter that can be approximately characterised by a single number.

The acceptable zone sizes specified in M42-A (CLSI, 2006a) for the A. salmonicida control strain incubated at $22 \pm 2{ }^{\circ} \mathrm{C}$ and read at $44-48 \mathrm{~h}$ are quite wide. For the discs used in this work they cover 11-14 mm (Table 1). The similarity, observed in this work, between the lateral shift in the epidemiological cut-off values and the lateral shift in the control strain means would suggest, therefore, that epidemiological cut-off values established by different laboratories, all in compliance with M42-A, could also vary over a similar, if slightly smaller, range. Even if the 'universal' cut-off value was established in a laboratory where the control strain mean was in the centre of the acceptable range, this cut-off value could be up to 5-6 mm distant from one that would be optimal for the data generated by another laboratory in full compliance with M42-A (CLSI, 2006a).

\subsection{The significance of lateral shift for the universal application of laboratory-independent cut-off values}

In order to discuss the significance of laboratoryspecific lateral shift for the application of universal epidemiological cut-off values it is useful to consider 
two types of error that might arise. Type 1 errors can arise when the universal cut-off values were generated in a laboratory where zone sizes are normally smaller than those in the laboratory applying them. Thus, type 1 errors would result in the laboratory applying the universal cut-off values classifying NWT strains as WT. The second (type 2) errors can occur when the universal cut-off values were generated in a laboratory where zone sizes are normally larger that in the laboratory applying them. Type 2 errors result in the classification of some WT strains as NWT.

In this work the lateral shift resulted in the epidemiological cut-off values estimated from Laboratory 1 data being, in all cases, smaller than those estimated from Laboratory 2 data (Tables 1 and 2). In two of the three case where a comparison can be made the Laboratory 1 cut-off values were also lower than those suggested by Miller and Reimschuessel (2006) (Table 2).

\subsubsection{Type 1 errors}

Type1 errors result in the failure to correctly identify strains with a NWT phenotype. In situations were a NWT phenotype results from the acquisition of a gene encoding a specific, positive function resistance (Sørum, 2006) thenit is reasonabletoassumethat the decreasein susceptibility would normally result in a large reduction in zone size (Uhland and Higgins, 2006). As a consequence, it is difficult to envisage a situation where the degree of lateral shift in the zone diameter data generated by a laboratory, would result in the failure to identify such strains.

Table2

The frequency of strains originally classified as wild type (WT) by Laboratory 1 that would not have been placed in this group if epidemiological cut-off values from other sources had been applied

\begin{tabular}{|c|c|c|c|c|}
\hline \multirow[t]{3}{*}{ Disc } & \multicolumn{3}{|c|}{ Epidemiological cut-off values } & \multirow{3}{*}{$\begin{array}{l}\text { Frequency } \\
\text { (\%) of type } \\
2 \text { errors }\end{array}$} \\
\hline & \multirow{2}{*}{$\begin{array}{l}\text { Lab } 1 \\
\text { Value }(\mathrm{mm}) \\
\end{array}$} & \multicolumn{2}{|c|}{ Alternative sources } & \\
\hline & & Source & Value $(\mathrm{mm})$ & \\
\hline ERY $15 \mu \mathrm{g}$ & $\geq 10$ & Lab 2 & $\geq 14$ & 0 \\
\hline \multirow[t]{2}{*}{ FLO $30 \mu \mathrm{g}$} & $\geq 27$ & Lab 2 & $\geq 31$ & $7 / 114(6 \%)$ \\
\hline & & M and $\mathrm{R} *$ & $\geq 31$ & $4(6 \%)$ \\
\hline GEN $10 \mu \mathrm{g}$ & $\geq 14$ & Lab 2 & $\geq 19$ & $3 / 105(3 \%)$ \\
\hline \multirow[t]{2}{*}{ OTC $30 \mu \mathrm{g}$} & $\geq 25$ & Lab 2 & $\geq 32$ ** & $13 / 56(23 \%)$ \\
\hline & & Mand R & $\geq 28$ & 0 \\
\hline \multirow[t]{2}{*}{ OXA $2 \mu \mathrm{g}$} & $\geq 30$ & Lab 2 & $\geq 33$ & $4 / 46(9 \%)$ \\
\hline & & Mand R & $\geq 30$ & 0 \\
\hline
\end{tabular}

* The source indicated as M and R was Miller and Reimschuessel (2006).

** Laboratory 2 was out of compliance with the acceptable ranges specified in M42-A for $\mathrm{OTC}_{30}$ discs.
However, there are data indicating that mechanisms other than the acquisition of specific genes can result in reduced susceptibility. Mechanisms such as multiple low-level resistance (Barnes et al., 1990, 1992; Griffiths and Lynch, 1989; Woods et al., 1986), efflux systems (Giraud et al., 2004; Poole, 2005), outer membrane changes (Nikaido, 1989) and even phenotypic, persistence mechanisms (Balaban et al., 2004; Levin, 2004) may result in much smaller changes in zone size. It is in the classification of strains manifesting low-level resistance that type 1 errors are most likely to occur.

The application of the cut-off values from Laboratory 1 or those of Miller and Reimschuessel (2006) to the data obtained in this work by Laboratory 2 would not have resulted in any type 1 errors. This absence of type 1 errors must be understood in the context of the rarity in the strain set studied of any strains that gave zones consistent with the possession of a low-level resistance mechanism. It should, however, be noted that other authors, for example Thyssen and Ollevier (2001) and Smith and Christofilogiannis (in press), have produced data that indicates a higher frequency of these mechanisms in the strain sets they studied. Smith et al. (1994) have suggested that some low-level resistance phenotypes, particularly with respect to quinolone agents, are unstable during storage. Thus, it is possible that the low frequency of strains manifesting low-level resistance might be related to the fact that the majority of strains studied in this work had been stored for a number of years.

\subsubsection{Type 2 errors}

Type 2 errors, caused by the application in a specific laboratory of an epidemiological cut-off value that is too large, result in the failure to correctly identify a strain as WT. In this work the cut-off values calculated by Laboratory 2 were, in all cases, larger than those calculated by Laboratory 1 . In addition two of the three cut-off values set by Miller and Reimschuessel (2006) were larger than those of Laboratory 1 . Table 2 presents the consequences that would have arisen if the data generated by Laboratory 1 had been interpreted using the cut-off values from Laboratory 2 or those suggested by Miller and Reimschuessel (2006). Laboratory 2 was, however, out of compliance with respect to OTC 30 , it would not have been legitimate, therefore, to attempt to generate an M42-A (CLSI, 2006a) applicable, laboratory-independent cut-off value from their data for this disc. Thus, the demonstration that the application by Laboratory 1 of the OTC cut-off values calculated by Laboratory 2 would have resulted in a miss-classification of $23 \%$ of the strains has only limited relevance. 
However, with respect to the other discs, the application of Laboratory 2 cut-off values to Laboratory 1 data would have resulted in type 2 errors occurring with a frequency of zero for $\mathrm{ERY}_{15}, 6 \%$ for $\mathrm{FLO}_{30}, 3 \%$ for $\mathrm{GEN}_{10}$ and $9 \%$ for OXA 2 . Application, by Laboratory 1, of the epidemiological cut-off value suggested by Miller and Reimschuessel (2006) for $\mathrm{FLO}_{30}$ would have resulted in type 2 errors with a frequency of $6 \%$.

These arguments demonstrate that the problems that lateral shift presents for the development of laboratoryindependent are not just theoretical and hypothetical.

\subsection{Application of NRI analysis}

Figs. 1a, 2a, 3a, 4a and 5a demonstrate that the application of laboratory specific cut-off values generated by NRI analysis resulted in a complete agreement between the two laboratories as to the classification (WT or NWT) of all strains. This agreement was achieved despite the existence of significant lateral shift(Table1) between the data generated by the two laboratories. This agreement was also achieved despite the failure of Laboratory 2 toobtain zonesforOTC 30 that were within the acceptable range for M42-A (CLSI, 2006a).

Compliance with the acceptable ranges for $\mathrm{OTC}_{30}$ has been a continuing problem for Laboratory 2 where supplementation of the Mueller-Hinton agar with divalent ions has been found to be necessary to achieve compliance with the specifications of M42-A (CLSI, 2006a). The approach of rigorous standardisation to the setting of cut-off values would require that, as the $\mathrm{OTC}_{30}$ data obtained by Laboratory 2 for the control strain was out of compliance with M42-A (CLSI, 2006a), the OTC $_{30}$ data for the other 106 strains should not be considered as having been generated by a legitimate application of M42-A (CLSI, 2006a). As a consequence, any epidemiological cut-off values established for this protocol should not be applied to the interpretation of $\mathrm{OTC}_{30}$ data from Laboratory 2. Equally, the $\mathrm{OTC}_{30}$ data from Laboratory 2 could not be used to generate cut-off values for data generated by this protocol. If the approach of rigorous standardisation and laboratory-independent cut-off values were adopted, Laboratory 2 would have to modify its test protocol, until it could achieve compliance with the acceptable ranges specified in M42-A (CLSI, 2006a), before it could interpret any of the data it generated from clinicalisolates. It is a clear advantage of the application of NRI analysis to setting laboratory-specific cut-off values, that it would allow Laboratory 2 to interpret their $\mathrm{OTC}_{30}$ data without modifying their performance of the test protocol.

\subsubsection{Ambiguity in classification of strains}

With respect to $\mathrm{GEN}_{10}$ and $\mathrm{OTC}_{30}$ the cut-off values set by each laboratory provided an unambiguous separation of strains on the basis of their zone sizes. With respect to $\mathrm{OXA}_{2}$ Laboratory 1 recorded one strain at its cut-off value. For the same strain Laboratory 2 recorded a zone size in the middle of the WT cluster suggesting that its classification as WT was probably correct. With respect to $\mathrm{FLO}_{30}$ there was, however, one strain that both laboratories placed close to their respective cut-off values (Fig. 2a). Miller and Reimschuessel (2006) set their disc diffusion epidemiological cut-off value for florfenicol such that $2 \%$ of their strains gave zones slightly below that value. The MIC values recorded for these strains did not, however, confirm their NWT status. Michel et al. (2005) reported that the presence of efflux pump inhibitors resulted in a $4 \mathrm{~mm}$ increase in zone size of $A$. salmonicida against $\mathrm{FLO}_{30}$ and this suggests that such pumps may mediate slight reduction in susceptibility to florfenicol in this strain. These observations suggest that continuing attention should be paid to strains that generate zones close to any cut-off value set for $\mathrm{FLO}_{30}$ discs.

\subsubsection{Patterns of susceptibility analysed by agent}

Although, with the exception of Norway (Lunestad and Grave, 2005) it is difficult to obtain quantitative data on the use of different antimicrobials in aquaculture, the frequency of NWT phenotypes to the different agents tested generally reflected the frequency with which they are believed to be employed in the control of furunculosis (Hastings, 1997). The very low frequencies of strains classified as NWT with respect to erythromycin and gentamicin are consistent with our understanding that

Table3

Distance between epidemiological cut-off values for WT strains and the means for control strain $(n=9)$ and the WT group mean calculated by NRI analysis

\begin{tabular}{llcc}
\hline Disc & Lab & \multicolumn{2}{c}{$\begin{array}{l}\text { Distance }(\mathrm{mm}) \text { of epidemiological } \\
\text { cut-off valuefrom }\end{array}$} \\
\cline { 2 - 4 } & & Control strain mean & WT mean \\
\hline ERY 15 $\mu \mathrm{g}$ & 1 & 11 & 9 \\
& 2 & 11 & 9 \\
FLO 30 $\mu \mathrm{g}$ & 1 & 9 & 9 \\
& 2 & 10 & 10 \\
GEN 10 $\mu \mathrm{g}$ & 1 & 8 & 8 \\
OTC 30 $\mu \mathrm{g}$ & 2 & 7 & 9 \\
& 1 & 7 & 9 \\
OXA 2 $\mu \mathrm{g}$ & 2 & 8 & 8 \\
& 1 & 5 & 8 \\
\hline
\end{tabular}


these agents are rarely, if ever, used to treat furunculosis in European aquaculture. In contrast, the relatively high frequencies of $\mathrm{OTC}_{\mathrm{NWT}}(48 \%)$ and $\mathrm{OXA}_{\mathrm{NWT}}(57 \%)$ phenotypes are consistent with the widespread use of these agents. With respect to florfenicol none of the European strains were classified as NWT. This may reflect the fact that many of the strains had been originally isolated before the widespread use of this agent in European aquaculture. However Michel et al. (2003) have reported that widespread use of florfenicol in France had not resulted in high frequencies of resistance in strains isolated in that country.

\subsubsection{Patterns of susceptibility analysed by country}

The strain set used in this work was composed of strains from 5 or, in the case of florfenicol, 6 countries. However, the various national collections were not isolated during the same time-period and were not composed of similar numbers of strains. Furthermore none of thenational strain setshad been selected as being representative of the $A$. salmonicida in that area. For these reasons no attempt has been made to provide an analysis of the distribution of NWT phenotypes by country.

\subsubsection{Similarity in the spread of zones for susceptible (WT) strains}

The NRI method allows the calculation of the mean and standard deviations of the real distribution of the zone sizes of fully susceptible(WT) strains. In this work there was a considerable agreements in these NRI estimated standard deviations for all five agents recorded by both laboratories. The 10 standard deviations calculated ranged from $2.5 \mathrm{~mm}$ to $3.8 \mathrm{~mm}$ with a mean of $3.4 \pm 0.4 \mathrm{~mm}$. As the NRI method calculates the epidemiological cut-off value from the mean and the standard deviation, the similarity of the standard deviations has the consequence that there is a remarkably similar distance between the NRI means and the epidemiological cut-off values for all agents in both laboratories (mean $\pm \mathrm{sd}, 8.6 \pm 0.8 \mathrm{~mm}$ ) (Table 3). The distance between the epidemiological cut-off values and the control strain means $(11 \mathrm{~mm}-5 \mathrm{~mm}$; median $8 \mathrm{~mm})$ shows a greater degree of variation.

\section{Conclusions}

This work has demonstrated that significant lateral shift can be expected between the disc diffusion data generated in different laboratories even when these laboratories generated control strain data that is in compliance with the specification of a standard protocol. It has also been demonstrated that the extent of the lateral shift is sufficient to lead to problems for the application of laboratory-independent epidemiological cut-off values.

The NRI method was demonstrated to provide a method of generating laboratory-specific epidemiological cut-off values. The two laboratories in this study obtained complete agreement in the categorisation of all strains with respect to 5 agents when they used cut-off values developed by the application of NRI analysis to their own data.

\section{Acknowledgements}

We acknowledge the support of our colleagues in providing the strains used in this work. French strains were supplied by the GDSAA (Groupement de Défense Sanitaire Aquacole d'Aquitaine, France) and the LD 40 (Laboratoire Départemental des Landes, France). The Norwegian strains were supplied by Henning Sørum (Department of Food Hygiene and Infectious Biology, Norwegian School of Veterinary Science, Oslo). The Scottish strains were supplied by David Bruno (The Marine Laboratory, Aberdeen). The Danish strains were supplied by Inger Dalsgaard (Danish Institute for Fisheries Research, Frederiksberg). The Canadian strains were supplied by Carl Uhland (Faculté Médecine Vétérinaire, Université de Montréal).

\section{References}

Alderman, D.J., Smith, P, 2001. Development of draft protocols of standard reference methods for antimicrobial agent susceptibility testing of bacteria associated with fish diseases. Aquaculture 196, 211-243.

Balaban, N.Q., Merrin, J., Chait, R., Kowalik, L., Leibler, S., 2004. Bacterial persistence as a phenotypic switch. Science 305, 1622-1625.

Barnes, A.C., Lewin, C.S., Hastings, T.S., Amyes, S.G., 1990. Crossresistance between oxytetracycline and oxolinic acid in Aeromonas salmonicida associated with alterations in outer membrane proteins. FEMS Microbiology Letters 60, 337-339.

Barnes, A.C., Lewin, C.S., Hastings, T.S., Amyes, S.G.B., 1992. Alterations in outer membrane proteins identified in a clinical strain of Aeromonas salmonicida subsp. salmonicida. Journal of Fish Diseases 15, 279-282.

CLSI, 2002. Development of in vitro susceptibility testing criteria and quality control parameters for veterinary antimicrobial agents. Approved guideline M37-A2. Clinical and Laboratory Standards Institute, Wayne, Pennsylvania.

CLSI, 2006a. Methods for antimicrobial disk susceptibility testing of bacteria isolated from aquatic animals. Approved guideline M42-A. Clinical and Laboratory Standards Institute, Wayne, Pennsylvania.

CLSI, 2006b. Methodsfor broth dilution susceptibility testing of bacteria isolated from aquatic animals. Approved guideline M49-A. Clinical and Laboratory Standards Institute, Wayne, Pennsylvania.

Coyne, R., Bergh, Ø., Samuelsen, O., Andersen, K., Lunestad, B.T., Nilsen, H., Dalsgaard, I., Smith, P., 2004a. Attempt to validate 
breakpoint MIC values estimated from pharmacokinetic data obtained during oxolinic acid therapy of winter ulcer disease in Atlantic salmon (Salmo salar). Aquaculture 238, 51-66.

Coyne, R., Samuelsen, O, Bergh, Ø., Andersen, K., Pursell, L, Dalsgaard, I., Smith, P., 2004b. On the validity of setting breakpoint minimum inhibition concentrations at one quarter of the plasma concentration achieved following oral administration of oxytetracycline. Aquaculture 239, 23-35.

Coyne, R , Smith, P.,Dalsgaard, I., Nilsen, H , Kongshaug, H., Bergh, Ø , Samuelsen, O B., 2006. Winter ulcer disease of post-smolt Atlantic salmon: an unsuitable case for treatment? Aquaculture 253, 171-178.

EUCAST, 2000. Terminology relating to methods for the determination of susceptibility of bacteria to antimicrobial agents. European Committee on Antimicrobial Susceptibility Testing Definitive document E. Def 1.2 May 2000. http://www.srga.org/Eucastwt/ eucastdefinitions.htm.

Forsberg, P., Petersson, A.C., Kronvall, G., 1985. Determination of species- and laboratory-related interpretive breakpoints for doxycycline susceptibility testing using single-strain regression analysis. Acta Pathologica, Microbiologica et Immunologica ScandinavicaSection B 93, 53-60.

Giraud, E., Blanc, G., Bouju-Albert, A., Weill, F.-X., Donnay-Moreno, C., 2004. Mechanisms of quinolone resistance and clonal relationship among Aeromonas salmonicida strains isolated from reared fish with furunculosis. Journal of Medical Microbiology 53, 895-901.

Griffiths, S.G., Lynch, W.H., 1989. Characterization of Aeromonas salmonicida mutants with low-level resistance to multiple antibiotics. Antimicrobial Agents Chemotherapy 33, 19-26.

Hastings, T.S, 1997. Chemotherapy of furunculosis. In: Bernoth, E.M., Ellis, A.E., Midtlyng, P., Olivier, G., Smith, P.(Eds.), Furunculosis Multidisciplinary fish disease research. Academic Press, London.

Huys, G., Cnockaert, M., Bartie, K., Oanh, D.T.H., Phuong, N.T., Somsiri, T., Chinabut, S., Yussoff, F.M., Shariff, M., Giacomini, M., Bertone, S., Swings, J., Teale, A., 2005. Intra- and interlaboratory performance of antibiotic disk-diffusion-susceptibility testing of bacterial control strains of relevance for monitoring aquaculture environments. Diseases of Aquatic Oganisms 66, 197-204.

Joneberg, J., Rylander, M., Galas, M.F., Carlos, C , Kronvall, G., 2003. Analysis of parameters and validation of method for normalized interpretation of antimicrobial resistance. International Journal of Antimicrobial Agents 21, 525-535.

Kahlmeter, G., Brown, D.F.J., Goldstein, F.W., MacGowan, A.P., Mouton, J.W., Österlund, A., Rodloff, A., Steinbakk, M., Urbaskova, P., Vatopoulos, A., 2003. European harmonization of MIC breakpoints for antimicrobial susceptibility testing of bacteria. Journal of Antimicrobial Chemotherapy 52, 145-148.

Kronvall, G., 2003. Determination of the real standard distribution of susceptible strains in zone histograms. International Journal of Antimicrobial Agents 22, 7-13.

Kronvall, G., Kahlmeter, G., Myhre, E., Galas, M.F , 2003. A new method for normalized interpretation of antimicrobial resistance from disk test results for comparative purposes. Clinical Microbiology and Infection 9, 120-132.

Kronvall, G., Ringertz, S , Karlson, I., Göransson, E., Dornbusch, K., 1988. Laboratory- and species-specific interpretive breakpoints for disc diffusion tests of chloramphenicol susceptibility of Haemophilus influenzae. Antimicrobial Agents and Chemotherapy 32, 1484-1489.

Kronvall,G ,1982. Analysis of asinglereferencestrainfor determination of gentamicin regression line constants and inhibition zone diameter breakpoints in quality control of disk diffusion antibiotic susceptibility testing. Journal of Clinical Microbiology 16, 784-793.

Kronvall, G., Ringertz, S., 1991. Antibiotic disk diffusion testing revisited. Single-strain regression analysis. APMIS 99, 295-306.

Levin, B.R., 2004. Non-inherited resistance to antibiotics. Science 305, 1578-1579.

Lunestad, B.T., Grave, K., 2005. Therapeutic agents in Norwegian aquaculture from 2000 to 2004 : usage and residue control. Bulletin of the European Association of Fish Pathologists 25, 284-290.

MacGowan, A.P., Wise, R., 2001. Establishing MIC breakpoints and the interpretation of in vitro susceptibility tests. Journal of Antimicrobial Chemotherapy 48 (Supp1. S1), 17-28.

Manninen, R., Eerola, E., Huovinen, P., 1995. Disk diffusion susceptibility tests; need for laboratory-specific breakpoints. Scandinavian Journal of Infectious Disease 27, 45-49.

Manninen, R., Leinonen, M., Huovinen, P., Nissinen, A., and the Finnish Group for Antimicrobial Resistance, 1998. Reliability of disc diffusion susceptibility testing of Streptococcus pnermoniae and adjustment of laboratory-specific breakpoints. Journal of Antimicrobial Chemotherapy 41, 19-26.

Michel, C., Kerouault, B., Martin, C., 2003. Chloramphenicol and florfenicol susceptibility of fish-pathogenic bacteria isolated in France: comparison of minimum inhibitory concentrations, using recommended provisory standards for fish bacteria. Journal of Applied Microbiology 95, 1008-1015.

Michel, C., Matte-Tailliez, O., Kerouault, B., Bernardet, J.-F., 2005. Resistance pattern and assessment of phenicol agents' minimum inhibitory concentration in multiple drug resistant Chryseobacterium isolates from fish and aquatic habitats. Journal of Applied Microbiology 99, 323-332.

Miller, R.A., Walker, R.D., Baya, A., Clemens, K., et al., 2003. Antimicrobial susceptibility testing of aquatic bacteria: quality control disk diffusion ranges for Escherichia coli ATCC 25922 and Aeromonas salmonicida subsp. salmonicida ATCC 33658 at 22 and $28^{\circ} \mathrm{C}$. Journal of Clinical Microbiology 41, 4318-4323.

Miller, A., Reimschuessel, R., 2006. Epidemiological cutoff values for antimicrobial agents against Aeromonas salmonicida isolates determined by frequency distributions of minimal inhibitory concentration and diameter of zone of inhibition data. American Journal of Veterinary Research 67, 1837-1843.

Nikaido, H., 1989. Outer membrane barrier as a mechanism of antimicrobial resistance. Antimicrobial Agents and Chemotherapy 33, 1831-1836.

NicGabhainn, S., Amedeo, M., Bergh, O., Dixon, B , Donachie, L., Carson, J., Coyne, R, Curtin, J, Dalsgaard, I., Maxwell, G., Smith, P., 2003. The precision and robustness of published protocols for disc diffusion assays of antimicrobial agent susceptibility: an inter-laboratory study. Aquaculture 240, 1-18.

Poole, K., 2005. Efflux-mediated antimicrobial resistance. Journal of Antimicrobial Chemotherapy 56, 20-51.

Samuelsen, O.B., 2006. Pharmacokinetics of quinolones in fish: a review. Aquaculture 255, 55-75.

Smith, P, 2001. Accuracy, precision and meaning of antimicrobial agent susceptibility testing of bacteria associated with fish diseases. Aquaculture 196, 253-266.

Smith, P.,2006. Breakpoints for disc diffusion susceptibility testing of bacteria associated with fish diseases: a review of current practice. Aquaculture 261, 1113-1121.

Smith, $\mathrm{P}, \mathrm{Christofilogiannis,} \mathrm{P}$, in press. Application of normalised resistance interpretation to the detection of multiple low level resistance in strains of Vibrio anguillartom obtained from Greek fish farms. Submitted to Aquaculture. 
Smith, P., Hiney, M.P., Samuelsen, O.B., 1994. Bacterial resistance to antimicrobial agents used in fish farming: a critical evaluation of method and meaning. Annual Review of Fish Diseases 4, 273-313.

Sørum, H., 2006. Antimicrobial drug resistance in fish pathogens. In: Aarestrup, Frank M. (Ed.), Antimicrobial resistance in bacteria of animal origin, chapter 13. ASM press, Washington D.C., pp. 213-238.

Thyssen, A., Ollevier, F., 2001. In vitro antimicrobial susceptibility of Photobacterium damselae subsp. piscicida to 15 different antimicrobial agents. Aquaculture 200, 256-269.
Wood, S.C., McCashion, R.N., Lynch, W.H, 1986. Multiple low-level antibiotic resistance in Aeromonas salmonicida. Antimicrobial Agents and Cheotherapy 29, 992-996.

Uhland, F.C., Higgins, R., 2006. Evaluation of the susceptibility of Aeromonas salmonicida to oxytetracycline and tetracycline using antimicrobial disk diffusion and dilution susceptibility tests. Aquaculture 257, 111-117. 